18,12

\title{
Бистабильность многостенных углеродных нанострубок, расположенных на плоских подложках
}

\author{
(C) А.В. Савин ${ }^{1,2}$, О.И. Савина ${ }^{2}$ \\ ${ }^{1}$ Федеральный исследовательский центр химической физики им. Н.Н. Семенова РАН, \\ Москва, Россия \\ ${ }^{2}$ Российский экономический университет им. Г.В. Плеханова, \\ Москва, Россия \\ E-mail: asavin@center.chph.ras.ru
}

Поступила в Редакцию 25 июня 2019 г.

В окончательной редакции 25 июня 2019 г.

Принята к публикации 2 июля 2019 г.

Исследованы стационарные состояния многостенных углеродных нанотрубок. Численное моделирование показало, что при достаточно больших диаметрах нанотрубки становятся бистабильными системами. Независимо от взаимодействия с подложками они могут находится в двух устойчивых состояниях: в открытом, имеющим внутреннюю полость, и в схлопнутом (коллапсированном) состоянии. Взаимодействие с плоской подложкой приводит к сплющиванию нанотрубки, делая энергетически более выгодным ее переход в схлопнутое состояние (чем сильнее взаимодействие, тем более выгодным становиться переход). Показано, что изменение формы многослойной нанотрубки из-за ее взаимодействия с подложкой или вследствие ее коллапса приводит к резкому увеличению числа ее коллективных собственных колебаний, в которых уже участвуют все ее слои. Получен энергетический профиль перехода между стационарными состояниями нанотрубки, который является сильно несимметричным двухъямным потенциалом, с первым узким минимумом, соответствующим схлопнутому состоянию, и со вторым широким минимумом, соответствующим открытому состоянию нанотрубки.

Ключевые слова: многостенная нанотрубка, бистабильность, коллапс нанотрубок, плоская подложка, двухъямный потенциал.

DOI: $10.21883 /$ FTT.2019.11.48438.535

\section{1. Введение}

Углеродные нанотрубки (УНТ) представляют собой цилиндрические макромолекулы диаметра от 0.4 нанометра и длиной до нескольких микрометров, состоящих из одного или нескольких слоев графена. Похожие структуры были получены 67 лет назад при термическом разложении окиси углерода на железном контакте [1]. Сами нанотрубки получены 28 лет назад как побочные продукты синтеза фуллерена $\mathrm{C}_{60}$ [2]. В настоящее время УНТ привлекают внимание благодаря своим уникальным свойствам [3].

Нанотрубки обладают высокой продольной (осевой) и относительно слабой поперечной (радиальной) жесткостью. В силу этого при достаточно больших диаметрах нанотрубки из-за невалентного взаимодействия ее слоев могут переходить из пустотелой цилиндрической формы в схлопнутое (коллапсированное) состояние [4-7]. Невалентное взаимодействие с подложкой тоже может изменять цилиндрическую форму нанотрубки [8-10].

До настоящего времени коллапс нанотрубок моделировался только с использованием либо полноатомных моделей $[7,11,12]$, либо континуальных моделей $[10,13,14]$. Такие модели не позволяют рассмотреть многослойные нанотрубки. В данной работе с использованием 2D цепной модели будут рассмотрены как изоли- рованные многослойные нанотрубки, так и нанотрубки, лежащие на плоских подложках (плоских поверхностях молекулярных кристаллов). Будет показано, что при достаточно больших диаметрах нанотрубки становятся бистабильными системами. Независимо от взаимодействия с подложками они могут находится в двух устойчивых состояниях: в открытом, имеющем внутреннюю полость, и в схлопнутом (коллапсированном) состоянии. Будет показано, что энергетический профиль перехода между стационарными состояниями является сильно несимметричным двухъямным потенциалом, с первым узким минимумом, соответствующим схлопнутому состоянию, и со вторым широким минимумом, соответствующим открытому состоянию нанотрубки.

\section{2. Модель многостенной углеродной нанотрубки на плоской подложке}

Для моделирования углеродных нанотрубок и нанолент, расположенных на плоских подложках, образованных поверхностями молекулярных кристаллов необходимо найти потенциал взаимодействия атома углерода c подложкой. С этой целью была найдена энергия взаимодействия конечного листа графена с плоской поверхностью кристалла. 


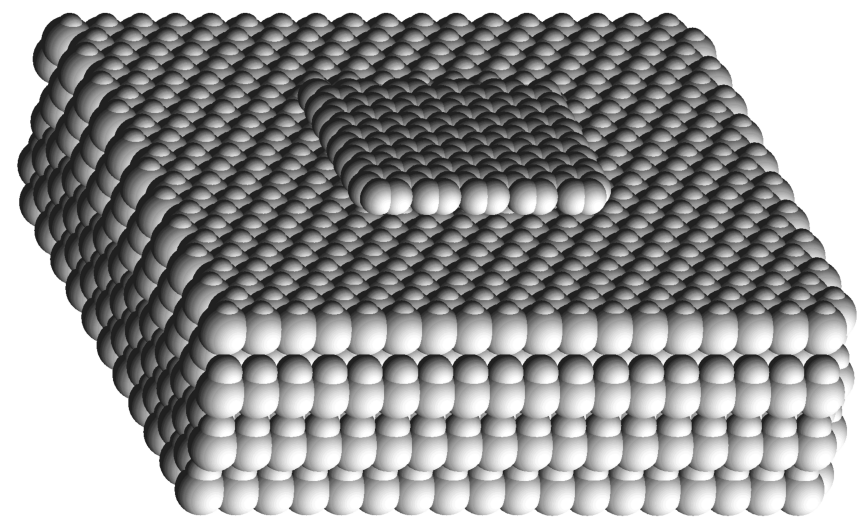

Рис. 1. Квадратный лист графена из 160 атомов, расположенный на плоской поверхности кристалла карбида кремния $6 H$-SiC [18]. К атомам кремния, образующим поверхность кристалла, присоединены атомы водорода. Показаны образцы, использованные при вычислении потенциала взаимодействия с подложкой $W_{0}(h)$.

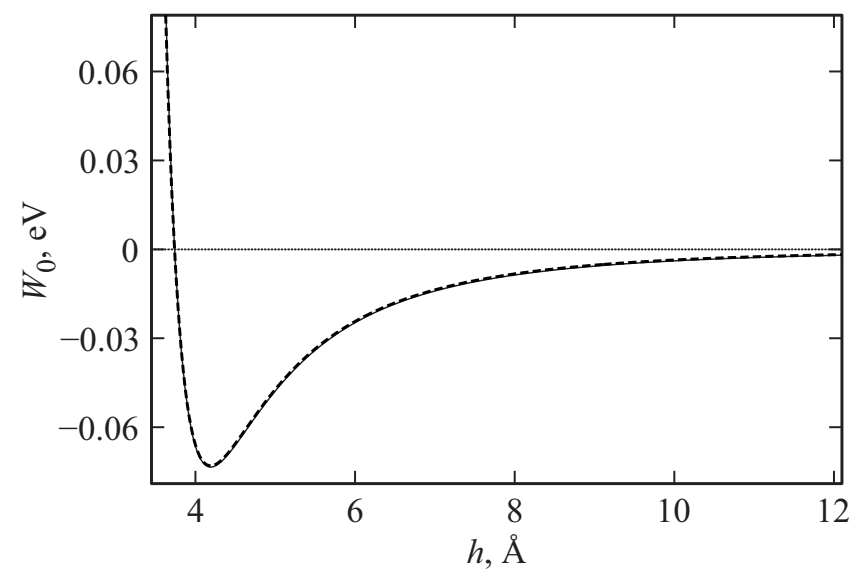

Рис. 2. Потенциал взаимодействия $W_{0}(h)$ атома углерода с плоской поверхностью кристалла карбида кремния 6H-SiC. Сплошная линия дает зависимость, полученную численно, пунктирная линия - зависимость, описываемую потенциалом Леннарда-Джонса (2) с параметрами $\epsilon_{1}=0.073 \mathrm{eV}$, $h_{1}=4.19 \AA, l=17, k=3.75$.

При вычислениях использовался лист графена размеpa $1.985 \times 1.843 \mathrm{~nm}^{2}$, состоящий из 160 атомов углерода, расположенный параллельно поверхности кристалла на расстоянии $h-$ см. рис. 1. При каждом значении расстояния $h$ энергия усреднялась по сдвигу по осям $x$ и $y$ на $2 \AA$ (усреднялась по квадрату $-1 \leq d x \leq 1 \AA$, $-1 \leq d y \leq 1 \AA$ ), а потом нормировалась на число атомов в листе графена. В результате получалась зависимость энергии взаимодействия с подложкой одного атома листа от его расстояния до плоскости подложки $W_{0}(h)$.

Энергия взаимодействия атомов листа графена с атомами молекулярного кристалла вычислялась с помощью потенциала Леннарда-Джонса

$$
V(r)=\epsilon\left[\left(r_{0} / r\right)^{12}-2\left(r_{0} / r\right)^{6}\right],
$$

где $\epsilon-$ энергия связи, $r_{0}$ - длина связи. Для нахождения энергии взаимодействия графена с поверхностью кристаллического графита использовались значения параметров потенциала из работы [15], а для остальных структур - из работ [16,17]. Использованные значения параметров потенциала (1) для различных пар атомов приведены в табл. 1 .

Вычисления показали, см. рис. 2, что энергия взаимодействия с подложкой $W_{0}(h)$ с высокой точность может быть описана $(k, l)$ потенциалом Леннарда-Джонсона

$$
W_{0}(h)=\epsilon_{0}\left[k\left(h_{0} / h\right)^{l}-l\left(h_{0} / h\right)^{k}\right] /(l-k),
$$

где степень $l>k$. Потенциал (2) имеет минимум $W_{0}\left(h_{0}\right)=-\epsilon_{0} \quad\left(\epsilon_{0}-\right.$ энергия связи атома с подложкой). Жесткость взаимодействия с подложкой $K_{0}=W^{\prime \prime}\left(h_{0}\right)=\epsilon_{0} l k / h_{0}^{2}$. Значения параметров потенциала (2) для различных кристаллов приведены в табл. 2.

При расположении графена на поверхности кристаллического никеля происходит более сильное химическое взаимодействие атомов углерода с атомами подложки. При моделировании это взаимодействие описывают суммой парных потенциалов Морса [19-21]. Поэтому взаимодействие атома углерода наноленты с поверхностью (111) кристалла Ni тоже удобно описать потенциалом Морса

$$
W_{0}(h)=\epsilon_{0}\left\{\exp \left[-\alpha\left(h-h_{0}\right)\right]-1\right\}^{2}-\epsilon_{0} .
$$

Энергия взаимодействия с поверхностью $\epsilon_{0}=$ $=0.133 \mathrm{eV}$ [22], равновесное расстояние до плоскости

Таблица 1. Значения параметров потенциала ЛеннардаДжонса (1) для различных пар взаимодействующих атомов. Второй набор параметров для атомов СС [15] использовался для атомов углерода, находящихся в плоских листах

\begin{tabular}{l|l|l|l}
\hline Атомы & $\epsilon,(\mathrm{eV})$ & $r_{0},(\AA)$ & Ссылка \\
\hline $\mathrm{CH}$ & 0.00295 & 3.369 & {$[16]$} \\
$\mathrm{CO}$ & 0.00344 & 3.676 & {$[16]$} \\
$\mathrm{CSi}$ & 0.00892 & 4.073 & {$[16]$} \\
$\mathrm{CC}$ & 0.00456 & 3.851 & {$[16]$} \\
$\mathrm{CC}$ & 0.00276 & 3.809 & {$[15]$} \\
$\mathrm{CAu}$ & 0.01273 & 2.9943 & {$[17]$}
\end{tabular}

Таблица 2. Значение параметров потенциала взаимодействия с плоской подложкой (2) для различных кристаллов

\begin{tabular}{l|l|l|l|l|c}
\hline \multicolumn{1}{c|}{ Атомы } & $\epsilon_{0},(\mathrm{eV})$ & $h_{0},(\AA)$ & $l$ & $k$ & $K_{0},(\mathrm{~N} / \mathrm{m})$ \\
\hline Лед $\mathrm{I}_{h}$ & 0.029 & 3.005 & 10 & 3.5 & 1.80 \\
Графит & 0.052 & 3.37 & 10 & 3.75 & 2.75 \\
Карбид кремния 6H-SiC & 0.073 & 4.19 & 17 & 3.75 & 4.24 \\
Поверхность $(111) \mathrm{Si}$ & 0.0564 & 4.09 & 16 & 3.75 & 3.24 \\
Поверхность (111) $\mathrm{Au}$ & 0.0732 & 2.96 & 10 & 3.5 & 4.68
\end{tabular}


подложки $h_{0}=2.145 \AA \quad[23] . \quad$ В результате взаимодействия листа графена с поверхностью кристалла внизу частотного спектра поперечных колебаний листа возникает щель величины $\omega_{0}=240 \mathrm{~cm}^{-1}$ [24]. Отсюда можно оценить коэффициент жесткости взаимодействия атома листа с подложкой $K_{0}=\omega_{0}^{2} M=41 \mathrm{~N} / \mathrm{m} \quad(M-$ масса атома углерода), а значит и значение параметра $\alpha=\sqrt{K_{0} / 2 \epsilon_{0}}=3.1 \AA^{-1}$.

Для описания многостенной углеродной нанотрубки удобно использовать двухмерную модель молекулярной цепи, позволяющую с высокой точностью описывать возможные стационарные состояния рулонных и складчатых упаковок нанолент графена [25,26]. Для одностенной углеродной нанотрубки со структурой зигзаг (с индексом хиральности $(m, 0))$ цепная модель описывает поперечное сечение нанотрубки, образующее кольцевую цепочку из $N=2 m$ эффективных частиц, описывающих продольные линии атомов нанотрубки.

Будем считать, что нанотрубка ориентирована вдоль оси $z$, тогда одной эффективной частице соответствуют все атомы нанотрубки, имеющие одинаковые координаты $x y$. Если эти атомы двигаются синхронно, меняя только координаты $x y$, но не меняя координату $z$, то гамильтониан нанотрубки можно записать как гамильтониан циклической цепи

$$
\begin{aligned}
H= & \sum_{n=1}^{N}\left[\frac{1}{2} M\left(\dot{x}_{n}^{2}+\dot{y}_{n}^{2}\right)+V\left(R_{n}\right)+U\left(\theta_{n}\right)\right. \\
& \left.+\frac{1}{2} \sum_{|k-n|>5} W_{1}\left(r_{n, k}\right)\right]
\end{aligned}
$$

где вектор $\mathbf{u}_{n}=\left(x_{n}, y_{n}\right)$ задает координаты $n$-ой частицы, $M$ - масса атома углерода.

Потенциал

$$
V(R)=\frac{1}{2} K(R-a)^{2}
$$

описывает продольную жесткость цепи, $K-$ жесткость взаимодействия, $a-$ равновесная длина связи (шаг цепи), $R_{n}=\left|\mathbf{u}_{n+1}-\mathbf{u}_{n}\right|$ - расстояние между соседними узлами $n$ и $n+1$.

Потенциал

$$
U(\theta)=\epsilon_{2}[1+\cos (\theta)]
$$

описывает изгибную жесткость цепи, $\theta-$ угол между двумя соседними связями, $\cos \left(\theta_{n}\right)=-\left(\mathbf{v}_{n-1}, \mathbf{v}_{n}\right) / R_{n-1} R_{n}$, вектор $\mathbf{v}_{n}=\mathbf{u}_{n+1}-\mathbf{u}_{n}$.

Потенциал $W_{1}\left(r_{n, k}\right)$ описывает слабые невалентные взаимодействия удаленных узлов цепи $n$ и $k$, $r_{n, k}=\left|\mathbf{u}_{k}-\mathbf{u}_{n}\right|$ - paсcтояние между узлами. Потенциал $W_{1}(r)$ может быть найден численно как среднее значение энергии взаимодействия атома углерода с координатами $\mathbf{v}_{0}=(r, y, 0)$ с цепочкой атомов $\left\{\mathbf{w}_{n}=\left(0, y_{n}, 0\right)\right\}_{n=-\infty}^{+\infty}$, где $y_{2 l}=3 r_{0} l, y_{2 l+1}=r_{0}+3 r_{0} l$,

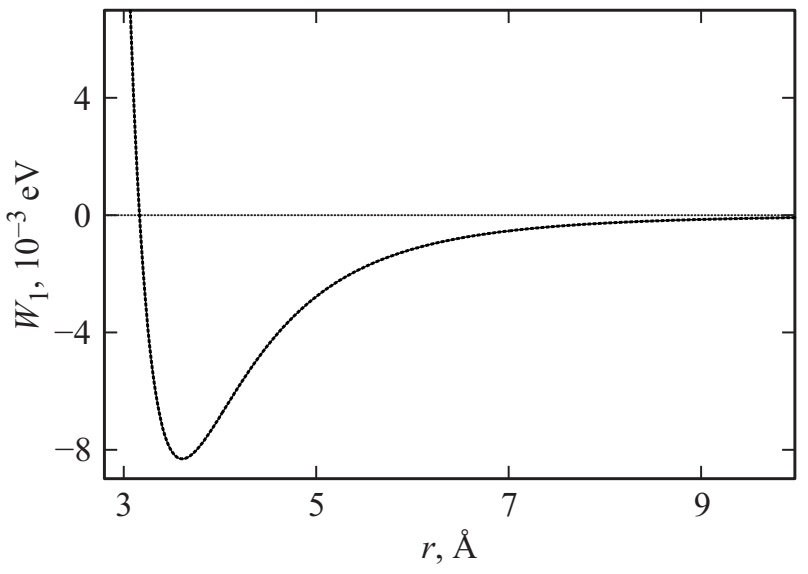

Рис. 3. Потенциал взаимодействия узлов цепи $W_{1}(r)$. Сплошная линия дает зависимость, полученную численно, пунктирная линия - зависимость, описываемую потенциалом Леннарда-Джонса (7).

$l=0, \pm 1, \pm 3, \ldots\left(r_{0}=1.418 \AA-\right.$ длина в листе графена валентной связи $\mathrm{C}-\mathrm{C})$ :

$$
W_{1}(r)=\frac{1}{3 r_{0}} \int_{0}^{3 r_{0}} \sum_{n=-\infty}^{+\infty} V_{C C}\left(r_{n}\right) d y, \quad r_{n}=\left|\mathbf{w}_{n}-\mathbf{v}_{0}\right| .
$$

Здесь $V_{C C}(r)$ - потенциал Леннарда-Джонса (1), описывающий невалентное взаимодействие двух атомов углерода листа графена [15].

Как видно из рис. 3, потенциал $W_{1}(r)$ с высокой точностью может быть описан потенциалом ЛеннардаДжонса (2) с показателями $l=11, k=5$, энергией $\epsilon_{1}=0.00832 \mathrm{eV}$ и равновесной длиной $r_{1}=3.607 \AA$. Таким образом, потенциал взаимодействия узлов цепи

$$
W_{1}(r)=\epsilon_{1}\left[5\left(r_{1} / r\right)^{11}-11\left(r_{1} / r\right)^{5}\right] / 6 .
$$

Параметры потенциалов (5), (6) определены в [25,26] из анализа дисперсионных кривых наноленты графена, продольная жесткость $K=405 \mathrm{~N} / \mathrm{m}$, шаг цепи $a=r_{0} \sqrt{3} / 2$, энергия $\epsilon_{2}=3.5 \mathrm{eV}$.

Многостенная ( $K$-слойная) нанотрубка

$$
\left(m_{1}, 0\right) @\left(m_{2}, 0\right) @ \ldots @\left(m_{K}, 0\right)=\left\{\left(m_{j}, 0\right)\right\}_{j=1}^{K}
$$

в цепной модели описывается как система вложенных друг в друга циклических цепочек - см. рис. 4, $a$. Рассмотрим многослойные нанотрубки, в которых каждый следующий слой образует плотную обмотку предыдущего. Для этого достаточно в качестве индекса следующего слоя $m_{j+1}$ взять максимальное значение, удовлетворяющее условию $R_{j+1}-R_{j}<3.4 \AA$, где $R_{j}=a / 2 \sin \left(\pi / 2 m_{j}\right)$ - радиус одностенной нанотрубки с индексом хиральности $\left(m_{j}, 0\right)$.

Гамильтониан $K$-слойной нанотрубки будет иметь вид

$$
H=\sum_{j=1}^{K} \sum_{n=1}^{N_{j}} \frac{1}{2} M\left(\dot{\mathbf{u}}_{n, j}, \dot{\mathbf{u}}_{n, j}\right)+E,
$$


где $N_{j}=2 m_{j}$ - число узлов $j$-той циклической цепи, $\mathbf{u}_{n, j}=\left(x_{n, j}, y_{n, j}\right)$ - вектор, задающий положение $n$-го узла цепи. Потенциальная энергия

$$
\begin{aligned}
E & =\sum_{j=1}^{K} \sum_{n=1}^{N_{j}}\left[V\left(R_{n, j}\right)+U\left(\theta_{n, j}\right)+\frac{1}{2} \sum_{|k-n|>5} W_{1}\left(r_{n, j ; k, j}\right)\right] \\
& +\sum_{j_{1}=1}^{K-1} \sum_{j_{2}=j_{1}+1}^{K} \sum_{n_{1}=1}^{N_{j_{1}}} \sum_{n_{2}=1}^{N_{j_{2}}} W_{1}\left(r_{n_{1}, j_{1} ; n_{2}, j_{2}}\right)+\sum_{j=1}^{K} \sum_{n=1}^{N_{j}} W_{0}\left(y_{n, j}\right),
\end{aligned}
$$

где первая сумма описывает энергию деформации всех цепочек (всех слоев нанотрубки), вторая - энергию межцепного взаимодействия (взаимодействия слоев), третья - энергию взаимодействия слоев нано-

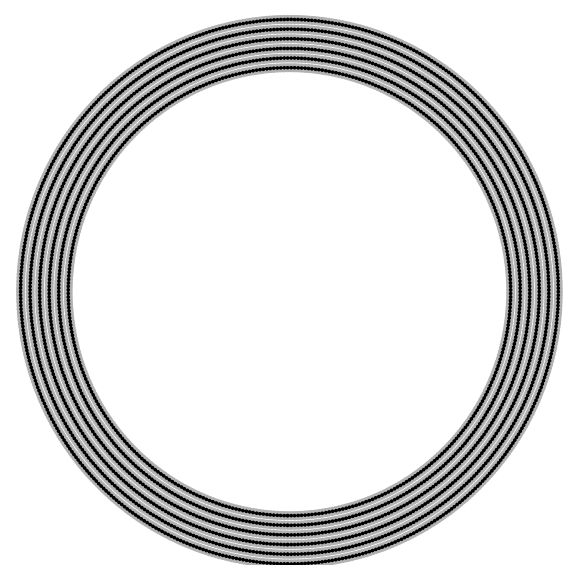

$a$

$b$
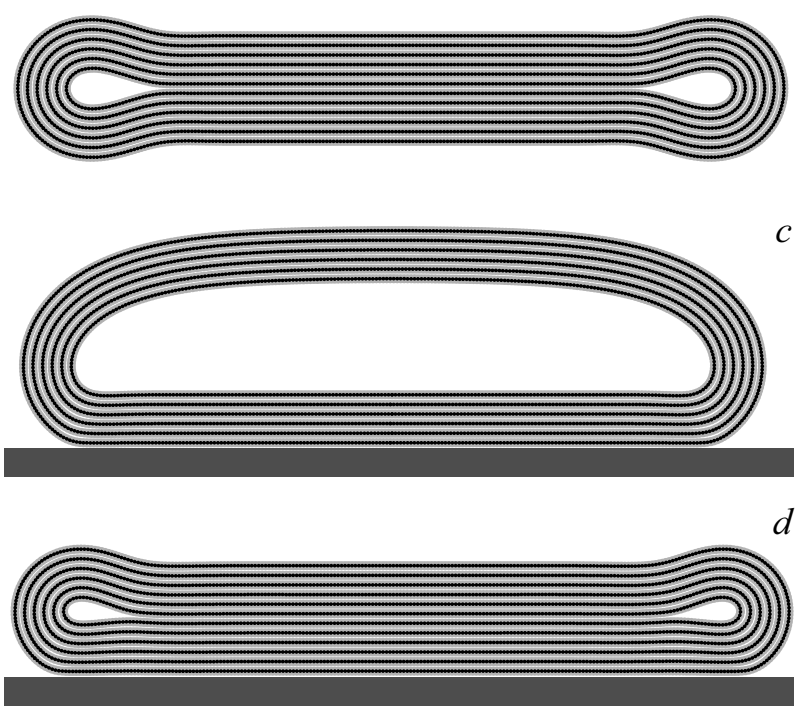

Рис. 4. Стационарные состояния шестистенной нанотрубки $\{(191+9 j, 0)\}_{j=1}^{6}:(a)$ открытое состояние с круговой симметрией (энергия $E=-111.5 \mathrm{eV}$, диаметры $D_{x}=D_{y}=190.6 \AA$ ) и $(b)$ схлопнутое состояние $\left(E=-114.8 \mathrm{eV}, D_{x}=271.6 \AA\right.$, $\left.D_{y}=48.1 \AA\right)$ изолированной нанотрубки; $(c)$ открытое каплеообразное состояние $\left(E=-125.0 \mathrm{eV}, \quad D_{x}=261.9 \AA\right.$, $\left.D_{y}=74.3 \AA\right)$ и $(d)$ схлопнутое состояние $(E=-131.7 \mathrm{eV}$, $\left.D_{x}=274.4 \AA, D_{y}=42.8 \AA\right)$ нанотрубки на плоской подложке карбида кремния $6 \mathrm{H}-\mathrm{SiC}$. трубки с плоской подложкой $y=-h_{0}$. Здесь расстояние $R_{n, j}=\left|\mathbf{v}_{n, j}\right|$, вектор $\mathbf{v}_{n, j}=\mathbf{u}_{n+1, j}-\mathbf{u}_{n, j}$, угол $\theta_{n, j}$ определяется из уравнения $\cos \left(\theta_{n, j}\right)=-\left(\mathbf{v}_{n-1, j}, \mathbf{v}_{n, j}\right)$ / $R_{n-1, j} R_{n, j}$, расстояние $r_{n_{1}, j_{1} ; n_{2}, j_{2}}=\left|\mathbf{u}_{n_{2}, j_{2}}-\mathbf{u}_{n_{1}, j_{1}}\right|$.

\section{3. Стационарные состояния нанотрубок}

Для нахождения стационарного состояния $K$-слойной нанотрубки $\left\{\left(m_{j}, 0\right)\right\}_{j=1}^{K}$ нужно решить задачу на минимум

$$
E \rightarrow \min :\left\{\mathbf{u}_{n, j}\right\}_{n=1, j=1}^{N_{j}, K} .
$$

Задача (10) решалась численно методом сопряженных градиентов. Выбирая начальную точку процедуры минимизации, можно получить все основные стационарные состояния многостенной нанотрубки - см. рис. 4. Изолированная нанотрубка имеет два стационарных состояния - открытое с круговой симметрией (a) и схлопнутое состояние, имеющее симметричную гантелеобразную форму (b) (для определенности будем считать, что сжатие нанотрубки происходит вдоль оси $y$ ). Нанотрубка на плоской подложке тоже имеет два стационарных состояния - открытое в форме выпуклой капли (c) и схлопнутое в форме несимметричной гантели $(\mathrm{d})$.

Стационарное состояние $K$-слойной нанотрубки $\left\{\left\{\left(x_{n, j}^{0}, y_{n, j}^{0}\right)\right\}_{n=1}^{N_{j}}\right\}_{j=1}^{K} \quad$ характеризуется энергией $E$, продольным и поперечными диаметрами

$$
D_{x}=\max _{n 1, n 2}\left|x_{n_{1}, K}^{0}-x_{n_{2}, K}^{0}\right|, \quad D_{y}=\max _{n 1, n 2}\left|y_{n_{1}, K}^{0}-y_{n_{2}, K}^{0}\right| .
$$

Рассматриваемые нанотрубки полностью определяются числом слоев $K$ и индексом первого внутреннего слоя $\left(m_{1}, 0\right)$. Нанотрубка с индексом $(5,0)$ имеет наименьший возможный диаметр $D_{x}=D_{y}=4.0 \AA$ [27], поэтому всегда будем брать индекс внутреннего слоя $m_{1} \geq 5$.

Зависимости нормированной энергии $E / m_{1}$ и поперечного диаметра $D_{y}$ от индекса $m_{1}$ для шестислойных $(K=6)$ нанотрубок на различных подложках представлены на рис. 5. Открытое состояние нанотрубки существует для всех значений индекса внутреннего слоя $m_{1} \geq 5$, схлопнутое состояние - только при превышение критического значения $m_{1} \geq m_{1}^{\prime}$. Критическое значение индекса зависит от числа слоев и от типа подложки. При числе слоев $K=6$ индекс $m_{1}^{\prime}=44$ для изолированной нанотрубки, $m_{1}^{\prime}=42$ и $m_{1}^{\prime}=41$ для нанотрубки на подложке карбида кремния и (111) Ni.

Для открытого состояния изолированной нанотрубки ее диаметры всегда совпадают $D_{x}=D_{y}=a / \sin \left(\pi / 2 m_{K}\right)$. C увеличением значения индекса внутреннего слоя $m_{1}$ диаметры будут увеличиваться пропорционально $m_{1}$. Для схлопнутого состояния поперечный диаметр всегда меньше продольного $D_{y}<D_{x}$. Продольный диаметр растет как линейная функция $m_{1}$, а поперечный диаметр будет оставаться постоянным - см. рис. 5, $b$. Для нанотрубок на подложке продольный диаметр тоже будет всегда увеличиваться пропорционально $m_{1}$. Поперечный 
диаметр открытого состояния будет медленно монотонно увеличиваться, а для закрытого состояния будет оставаться постоянным.

При малых значениях индекса $m_{1}$ открытое состояние нанотрубки является более энергетически выгодным,



Рис. 5. Зависимость $(a)$ энергии $E / m_{1}$ и $(b)$ вертикального диаметра $D_{y}$ для шестистенной нанотрубки $\left\{\left(m_{j}, 0\right)\right\}_{j=1}^{6}$ от значения индекса внутреннего слоя $m_{1}$. Кривые 1,2 и 3, 4 дают зависимости для открытого и схлопнутого состояний изолированной нанотрубки; кривые 5,6 и 7,8 - для нанотрубки на плоской подложке карбида кремния $6 \mathrm{H}-\mathrm{SiC}$; кривые 9, 10 и 11,12 - для нанотрубки на поверхности (111) кристалла $\mathrm{Ni}$.

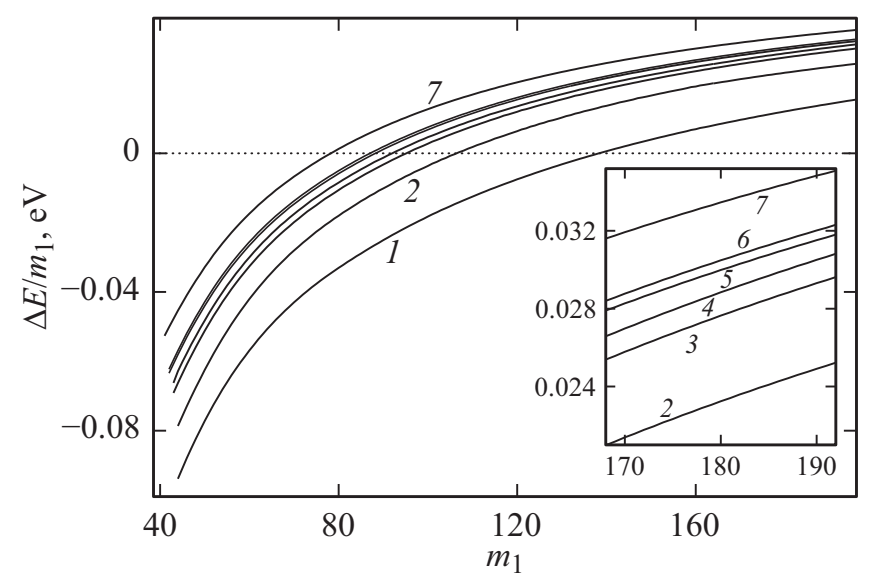

Рис. 6. Зависимость разницы энергии $\Delta E / m_{1}$ открытого и закрытого состояний шестистенной нанотрубки от значения индекса внутреннего слоя $m_{1}$ для изолированной нанотрубки (кривая 1), для нанотрубки на плоской поверхности льда, графита, на (111) поверхности кристаллов $\mathrm{Si}, \mathrm{Au}, 6 H-\mathrm{SiC}$ и $\mathrm{Ni}$ (кривые 2, 3, 4, 5, 6 и 7).
Таблица 3. Критические значения индексов внутреннего слоя $m_{1}^{\prime}, m_{1}^{\prime \prime}$ для $K$-слойных нанотрубок, лежащих на разных плоских подложках

\begin{tabular}{l|c|c|c|c|c|c}
\hline \multicolumn{1}{c|}{$K$} & 1 & 2 & 3 & 4 & 5 & 6 \\
\hline Без подложки & 33,81 & 36,96 & 39,110 & 40,122 & 42,131 & 44,138 \\
Лед $\mathrm{I}_{h}$ & 32,63 & 36,76 & 39,86 & 42,95 & 43,101 & 44,106 \\
Графит & 31,56 & 35,68 & 38,78 & 41,85 & 42,90 & 43,95 \\
$(111) \mathrm{Si}$ & 30,54 & 35,66 & 38,76 & 40,82 & 42,88 & 43,92 \\
6H-SiC & 29,50 & 34,62 & 37,71 & 39,78 & 41,83 & 42,87 \\
$(111) \mathrm{Au}$ & 30,52 & 34,64 & 38,73 & 40,79 & 41,84 & 42,88 \\
$(111) \mathrm{Ni}$ & 32,49 & 34,59 & 36,65 & 38,71 & 40,75 & 41,79
\end{tabular}

чем схлопнутое. С увеличением $m_{1}$ энергия открытого состояния растет быстрее энергии схлопнутого состояния. Существует второе критическое значение индекса $m_{1}^{\prime \prime}$, после которого схлопнутое состояние становится более энергетически выгодным - см. рис. 5, $a$. При числе слоев $K=6$ индекс $m_{1}^{\prime \prime}=138$ для изолированной нанотрубки, $m_{1}^{\prime \prime}=87$ и $m_{1}^{\prime \prime}=79$ для нанотрубки на подложке карбида кремния и (111) Ni.

Полученные критические значения индексов $m_{1}^{\prime}, m_{2}^{\prime \prime}$ для многослойных нанотрубок, лежащих на разных подложках, приведены в табл. 3. Как видно из табл, 2 и 3, чем сильнее взаимодействие с подложкой, тем меньше значения индексов $m_{1}^{\prime}$ и $m_{1}^{\prime \prime}$. Зависимость нормированной на $m_{1}$ разницы энергии открытого и схлопнутого состояний $\Delta E=E_{o}-E_{c}\left(E_{o}\right.$ и $E_{c}-$ энергия открытого и схлопнутого стационарного состояния нанотрубки) представлена на рис. 6. Разницу энергий можно определить только для $m_{1} \geq m_{1}^{\prime}$, когда существуют одновременно оба состояния. Как видно из рисунка, $\Delta E$ монотонно растет с увеличением значения индекса $m_{1}$. При $m_{1}<m_{1}^{\prime \prime}$ разница $\Delta E<0$, при $m_{1} \geq m_{1}^{\prime \prime} \Delta E \geq 0$. Разница энергии также монотонно увеличивается с увеличением энергии взаимодействия с подложкой. Таким образом, взаимодействие с подложкой приводит к сплющиванию нанотрубки, делая тем самым более энергетически выгодным ее переход в схлопнутое состояние.

\section{4. Частотный спектр стационарных состояний}

Для проверки устойчивости стационарных состояний многослойных нанотрубок был проведен анализ их малоамплитудных колебаний. Для этого были найдены все собственные значения и соответствующие собственные вектора матрицы вторых производных размерности $2 N_{a} \times 2 N_{a}$

$$
\mathbf{B}=\left(\left.\frac{\partial E}{\partial \mathbf{u}_{n_{1}, j_{1}} \partial \mathbf{u}_{n_{2}, j_{2}}}\right|_{\left\{\mathbf{u}_{n, j}^{0}\right\}}\right)_{n_{1}=1, j_{1}=1, n_{2}=1, j_{2}=1}^{N_{j_{1}}, K, N_{j_{2}}, K},
$$

где общее число узлов цепей $N_{a}=N_{1}+\ldots+N_{K}$, $\left\{\mathbf{u}_{n, j}^{0}=\left(x_{n, j}^{0}, y_{n, j}^{0}\right)\right\}_{n=1, j=1}^{N_{j}, K}-$ стационарное состояние $K$-слойной нанотрубки. 


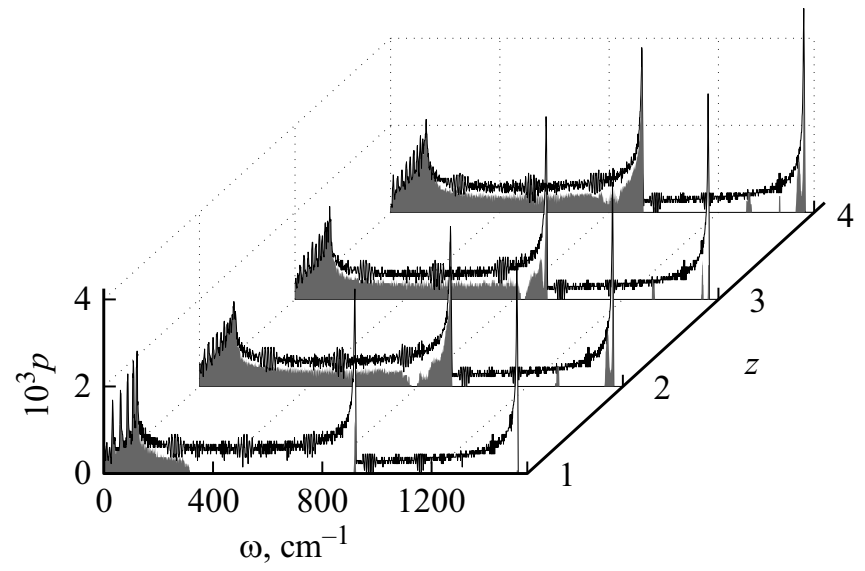

Рис. 7. Вид плотности частотного спектра $p(\omega)$ малоамплитудных колебаний шестистенной углеродной нанотрубки $\{(191+9 j, 0)\}_{j=1}^{6}$ для открытого $(z=1)$ и схлопнутого $(z=2)$ стационарного состояния изолированной нанотрубки, для открытого $(z=3)$ и схлопнутого $(z=4)$ стационарного состояния нанотрубки на плоской подложке карбида кремния $6 H$-SiC. Серым цветом показана плотность для коллективных колебаний, в которых принимают участие несколько слоев.

Стационарное состояние системы цепей будет устойчивым, только если все собственные значения симметричной матрицы В будут неотрицательными: $\lambda_{i} \geq 0$, $i=1,2, \ldots, 2 N_{a}$. Для устойчивой конфигурации первое собственное значение всегда будет равно нулю - этому значению соответствует сдвиг системы как твердого тела вдоль оси $x$. Остальным положительным собственным значениям соответствуют собственные колебания структуры с частотами $\omega_{i}=\sqrt{\lambda_{i+1} / M}, i=1, \ldots, 2 N_{a}-1$.

Анализ частотного спектра собственных колебаний показал, что открытые и схлопнутые стационарные состояния многослойных нанотрубок всегда являются устойчивыми (все собственные значения $\lambda_{i} \geq 0$ ). Схлопывание нанотрубок и их взаимодействие с подложками приводит только к малозаметному изменению плотности частотного спектра в области низких частот $\omega<200 \mathrm{~cm}^{-1}$ (см. рис. 7), но характер колебаний при этом может существенно меняться.

Пусть $\left\{\mathbf{e}_{n, j}\right\}_{n=1, j=1}^{N_{j}, K}-$ нормированный к единице собственный вектор, соответствующий собственной частоте $\omega_{i}$ :

$$
\sum_{l=1}^{K} p_{j}=\sum_{j=1}^{K} \sum_{n=1}^{N_{j}}\left|\mathbf{e}_{n, j}\right|^{2}=1,
$$

где двумерный вектор $\mathbf{e}_{n, j}$ описывает колебания узла $(n, j)$ системы $K$ цепей, а число $p_{j} \in[0,1]$ определяет долю энергии собственного колебания, приходящегося на $j$-ую цепь системы. Будем считать колебание коллективным, если доля колебаний каждой цепи не превосходит $90 \%\left(\max _{j} p_{j}<0.9\right)$, и изолированным в обратном случае. В коллективном колебании принимают участие сразу несколько цепей, а в изолированном участвует, в основном, только одна цепь.
Анализ собственных колебаний показывает, что для открытого стационарного состояния изолированной многостенной нанотрубки практически все собственные колебания с частотами $\omega>300 \mathrm{~cm}^{-1}$ являются изолированными - см. рис. 7. Здесь цилиндрическая симметрия состояния нанотрубки приводит к изоляции высокочастотных колебаний на ее отдельных слоях. Так для шестистенной нанотрубки $\{(191+9 j, 0)\}_{j=1}^{6}$ на коллективные (совместные) колебания слоев приходится только $p_{c}=0.214$ часть всех колебаний. Схлопывание нанотрубки, а также ее взаимодействие с подложкой приводит к исчезновению цилиндрической симметрии. В результате этого в спектре собственных колебаний резко возрастает доля совместных колебаний слоев: так $p_{c}=0.516$ для схлопнутого состояния изолированной нанотрубки, $p_{c}=0.485$ для открытого состояния и $p_{c}=0.569$ для схлопнутого состояния нанотрубки на подложке из карбида кремния. Как видно из рисунка, коллективными становится даже часть высокочастотных колебаний.

\section{5. Энергетический профиль между стационарными состояниями нанотрубки}

Полученные результаты позволяют заключить, что многостенная нанотрубка является бистабильной системой с двумя устойчивыми (открытым и схлопнутым) состояниями. Для нахождения энергетического рельефа перехода между этими состояниями нужно численно решить задачу на минимум (10) при каждом фиксированном значении расстояния $h$ между подложкой и центром внешней стороны внешнего слоя нанотрубки. Численное решение этой задачи позволяет получить зависимость $E(h)$, описывающую изменение энергии нанотрубки при ее схлопывании.

Вид потенциала $E(h)$ при разных значениях индекса внутреннего слоя нанотрубки $m_{1}$ представлен на рис. 8. Форма потенциала подтверждает полученные ранее результаты. Как видно из рисунка, при значении индекса первого слоя $m_{1} \leq m_{1}^{\prime}$ функция $E(h)$ является одноямным потенциалом с минимумом, соответствующим открытому состоянию. При $m_{1}>m_{1}^{\prime}$ потенциал становится двухъямным - появляется новый узкий минимум, соответствующий схлопнутому стационарному состоянию. С увеличением индекса $m_{1}$ (с увеличением радиуса) нанотрубки глубина нового узкого минимума монотонно увеличивается. При $m_{1}>m_{1}^{\prime \prime}$ этот минимум становится основным по энергии. Здесь энергетический профиль $E(h)$ имеет вид сильно асимметричного двухъямного потенциала с узким основным и широким вторым минимумом.

Характерная форма сильно несимметричного двухъямного потенциала $E(h)$ позволяет качественно объяснить результаты работы [28], где было показано, что тепловые колебания одностенной УНТ могут приводить 

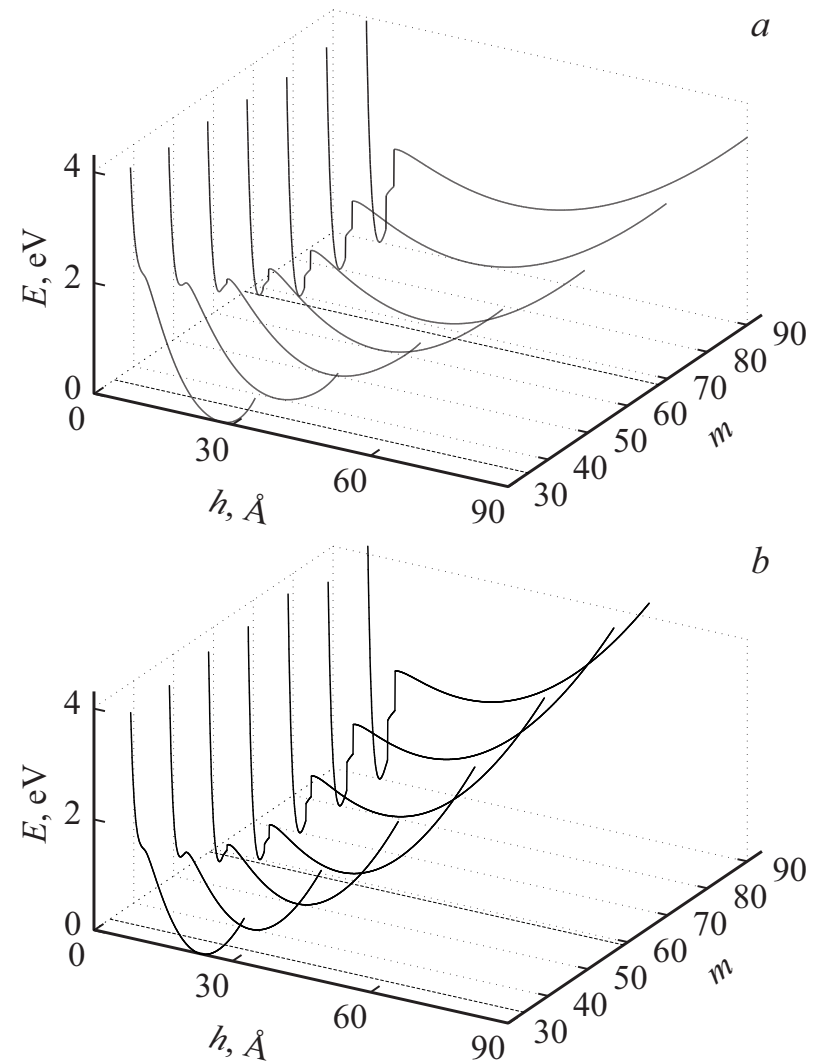

Рис. 8. Зависимость энергии $E$ двустенной нанотрубки $(m, 0) @(m+9.0)$, расположенной на подложке $(a)$ кристаллического графита и $(b)$ на поверхности (111) кристалла Ni от расстояния $h$ от подложки до противоположной стороны нанотрубки. Энергия отсчитывается от минимального значения. Пунктирные линии показывают критические значения индекса внутреннего слоя $m^{\prime}$ и $m^{\prime \prime}$.

к обращению ее перехода из открытого состояния в схлопнутое. Переход нанотрубки из одного стационарного состояния в другое качественно можно описать как движение кинка (топологического солитона) в модели $\phi-4$ с несимметричным двухъямным потенциалом $E(h)$, имеющим одну узкую глубокую яму, соответствующую схлопнутому состоянию, и более высокую по энергии вторую широкую яму, соответствующую открытому состоянию нанотрубки. Этот случай детально рассмотрен в работах $[29,30]$, где показано, что направление движения кинка в такой цепи зависит от значения температуры.

\section{6. Заключение}

Проведенное численное моделирование показывает, что одностенные и многостенные УНТ достаточно большого диаметра являются бистабильными системами. Нанотрубки могут находиться в двух устойчивых состояниях: в открытом, имеющим пустую внутреннюю полость, и в схлопнутом (коллапсированном) состо- янии. Бистабильностью обладают как изолированные, так и нанотрубки, находящиеся на плоской поверхности кристалла. Взаимодействие с подложкой приводит к сплющиванию нанотрубки, делая энергетически более выгодным ее переход в схлопнутое состояние (чем сильнее взаимодействие, тем более выгодным становится переход).

Изолированная нанотрубка имеет форму многослойного цилиндра. Из-за высокой симметрии этой формы основная часть собственных колебаний нанотрубки изолируется на ее отдельных слоях. Сплющивание нанотрубки из-за ее взаимодействия с подложкой или вследствие ее схлопывания приводит к тому, что большая часть ее собственных колебаний становится коллективными, в них начинают участвовать все слои.

Показано, что энергетический профиль перехода между двумя стационарными состояниями нанотрубки описывается сильно несимметричным двухъямным потенциалом, с первым узким минимумом, соответствующим схлопнутому состоянию, и со вторым широким минимумом, соответствующим открытому состоянию нанотрубки. Форма потенциала позволяет объяснить особенности динамики схлопывания одностенных нанотрубок.

\section{Финансирование работы}

Исследование выполнено при финансовой поддержке Российского научного фонда (проект 16-13-10302). Вычислительные ресурсы предоставлены межведомственным суперкомпьютерным центром РАН.

\section{Конфликт интересов}

Конфликт интересов: авторы заявляют об отсутствии конфликта интересов.

\section{Список литературы}

[1] Л.В. Радушкевич, В.М. Лукьянович. ЖФХ 26, 1, 88 (1952).

[2] S. Iijima. Nature 354, 568 (1991).

[3] А.В. Елецкий. УФН 172, 4, 401 (2002).

[4] N.S. Chopra, L.X. Benedict, V.H. Crespi, M.L. Cohen, S.G. Louie, A. Zettl. Nature 377, 14, 135 (1995).

[5] G. Gao, T. Çağin, W.A. Goddard. Nanotechnology 9, 184 (1998).

[6] J. Xiao, B. Liu, Y. Huang, J. Zuo, K.-C. Hwang, M.-F. Yu. Nanotechnology 18, 395703 (2007).

[7] J.A. Baimova, Q. Fan, L. Zeng, Z. Wang, S.V. Dmitriev, X. Feng, K. Zhou. J. Nanomater. 2015, 186231 (2015).

[8] T. Hertel, R.E. Walkup, P. Avouris. Phys. Rev. B 58, 20, 13870 (1998).

[9] J. Xie, Q. Xue, H. Chen, D. Xia, C. Lv, M. Ma. J. Phys. Chem. C 114, 2100 (2010).

[10] X. Yuan, Y. Wang. Nanotechnology 29, 075705 (2018).

[11] J. Kou, X. Zhou, Y. Fang, Y. Chen, H. Lu, H. Ye, F. Wu, J. Fan. Appl. Phys. Lett. 102, 123902 (2013).

[12] Y. Umeno, Y. Yachi, M. Sato, H. Shima. Physica E 106319 (2019). 
[13] T. Tang, A. Jagota, C.-Y. Hui, N.J. Glassmaker. J. Appl. Phys. 97, 074310 (2005).

[14] J. Liu. Arch. Appl. Mech. 82, 767 (2012).

[15] R. Setton. Carbon 34, 69 (1996).

[16] A.K. Rappe, C.J. Casewit, K.S. Colwell, W.A. Goddard III, W.M. Skiff. J. Am. Chem. Soc. 114, 10024 (1992).

[17] W.D. Luedtke, Uzi Landman. Phys. Rev. Lett. 82, 3835 (1999).

[18] J. Sforzini, L. Nemec, T. Denig, B. tadtmuller, T.-L. Lee, C. Kumpf, S. Soubatch, U. Starke, P.Rinke, V. Blum, F.C. Bocquet, F.S. Tautz. Phys. Rev. Lett. 114, 106804 (2015).

[19] A. Lyalin, A. Hussien, A.V. Solov'yov, W. Greiner. Phys. Rev. B 79, 165403 (2009).

[20] А.Е. Галашев, В.А. Полухин. ФТТ 55, 11, 2250 (2013).

[21] K.P. Katin, V.S. Prudkovskiy, M.M. Maslov. Micro Nano Lett. 13, 2, 160 (2018).

[22] J. Lahiri, T.S. Miller, A.J. Ross, L. Adamska, I.I. Oleynik, M. Batzill. New J. Phys. 13025001 (2011).

[23] Y. Gamo, A. Nagashima, M. Wakabayashi, M. Terai, C. Oshima. Surf. Sci. 374, 61 (1997).

[24] A. Dahal, M. Batzill. Nanoscale 6, 2548 (2014).

[25] A.V. Savin, E.A. Korznikova, S.V. Dmitriev. Phys. Rev. B 92 , 035412 (2015).

[26] А.В. Савин, Е.А. Корзникова, С.В. Дмитриев. ФТТ 57, 11, 2278 (2015).

[27] H.Y. Peng, N. Wang, Y.F. Zheng, Y. Lifshitz, J. Kulik, R.Q. Zhang, C.S. Lee, S.T. Lee. Appl. Phys. Lett. 77, 18, 2831 (2000).

[28] T. Chang, Z. Guo. Nano Lett. 10, 3490 (2010).

[29] A.V. Savin, G.P. Tsironis, A.V. Zolotaryuk. Phys. Rev. E, 56, 3, 2457 (1997)

[30] G. Costantini, F. Marchesoni. Phys. Rev. Lett. 87, 11, 114102 (2001).

Редактор К.В. Емцев 